\title{
Influence of ionization degree on film properties when using high power impulse magnetron sputtering
}

\author{
Mattias Samuelsson, Daniel Lundin, Kostas Sarakinos, Fredrik Bjorefors, Bengt Walivaara, \\ Henrik Ljungcrantz and Ulf Helmersson
}

\section{Linköping University Post Print}

\begin{abstract}
N.B.: When citing this work, cite the original article.
\end{abstract}
Original Publication:

Mattias Samuelsson, Daniel Lundin, Kostas Sarakinos, Fredrik Bjorefors, Bengt Walivaara, Henrik Ljungcrantz and Ulf Helmersson, Influence of ionization degree on film properties when using high power impulse magnetron sputtering, 2012, Journal of Vacuum Science \&amp; Technology. A. Vacuum, Surfaces, and Films, (30), 3, 031507.

http://dx.doi.org/10.1116/1.3700227

Copyright: American Vacuum Society http://www.avs.org/

This article may be downloaded for personal use only. Any other use requires prior permission of the author and the American Vacuum Society

Postprint available at: Linköping University Electronic Press http://urn.kb.se/resolve?urn=urn:nbn:se:liu:diva-78582 


\title{
Influence of ionization degree on film properties when using high power impulse magnetron sputtering
}

\author{
Mattias Samuelsson ${ }^{\text {a) }}$ \\ Plasma \& Coatings Physics Division, IFM-Materials Physics, Linköping University, SE-581 83 Linköping, \\ Sweden and Impact Coatings, Westmansgatan 29, SE-582 16 Linköping, Sweden \\ Daniel Lundin and Kostas Sarakinos \\ Plasma \& Coatings Physics Division, IFM-Materials Physics, Linköping University, SE-581 83 Linköping, \\ Sweden \\ Fredrik Björefors \\ Department of Materials Chemistry, The Angström Laboratory, Uppsala University, Box 538, SE-751 21 \\ Uppsala, Sweden
}

Bengt Wälivaara and Henrik Ljungcrantz

Impact Coatings, Westmansgatan 29, SE-582 16 Linköping, Sweden

U. Helmersson

Plasma \& Coatings Physics Division, IFM-Materials Physics, Linköping University, SE-581 83 Linköping, Sweden

(Received 23 November 2011; accepted 17 March 2012; published 3 April 2012)

\begin{abstract}
Chromium thin films are deposited by combining direct current magnetron sputtering and high power impulse magnetron sputtering (HiPIMS) on a single cathode in an industrial deposition system. While maintaining a constant deposition rate and unchanged metal ion energy distribution function, the fraction of the total power supplied by either deposition technique is altered, and thereby also the metal ion to metal neutral ratio of the deposition flux. It is observed that the required total average power needed to be proportionally increased as the HiPIMS fraction is increased to be able to keep a constant deposition rate. The influence on microstructure, electrical, and electrochemical properties of the films is investigated and shows improvements with the use of HiPIMS. However, considerable influence of the studied properties occurs already when only some $40 \%$ of the total power is supplied by the HiPIMS technique. Further increase of the HiPIMS power fraction results in comparatively minor influence of the studied properties yet significant deposition rate efficiency reduction. The results show that the degree of ionization can be controlled separately, and that the advantages associated with using HiPIMS can be obtained while much of the deposition rate reduction, often reported for HiPIMS, can be avoided. (C) 2012 American Vacuum Society. [http://dx.doi.org/10.1116/1.3700227]
\end{abstract}

\section{INTRODUCTION}

In high power impulse magnetron sputtering (HiPIMS) the power is applied to a conventional cathode in short unipolar pulses at low duty factors. ${ }^{1-3}$ By doing this, the instantaneous power density on the target and the plasma density is increased by approximately $2-3$ orders of magnitude as compared to direct current magnetron sputtering (DCMS). The higher plasma density, in turn, leads to a higher probability to ionize the sputtered vapor. ${ }^{4}$ A partially ionized deposition flux has been shown to allow for increased possibility in controlling the microstructure, phases, and chemical composition of the growing films, which may lead to enhanced mechanical, optical, and electrical film properties. ${ }^{3}$ Besides the beneficial influence of the HiPIMS process for the film properties, the power normalized deposition rates for HiPIMS are often observed to be lower than those for DCMS. ${ }^{5}$ For example, in the case of $\mathrm{Cr}$, as low as $30 \%$ of the DCMS rates have been reported. ${ }^{2}$ The reduction in deposition rate has been suggested to originate from the back

$\overline{{ }^{a} \text { Electronic mail: matsa757@ifm.liu.se }}$ attraction of ionized sputtered atoms. ${ }^{6}$ Other factors, such as changes in, and efficiency of, the transport mechanisms of ionized species through the bulk of the plasma toward the substrate, have also been proposed to contribute to the deposition rate loss. ${ }^{7-9}$

The HiPIMS process entails a wide parameter range, generating a variety of plasma conditions. ${ }^{10}$ By tuning the process parameters, such as peak target power or current, it is possible to compass plasma conditions ranging from DCMS-like to highly ionized ones. This was demonstrated by, e.g., Alami et al. ${ }^{11}$ who found that a change in ionization influenced the plasma impedance, and through this also the deposition rate. Although studies have shown that the degree of ionization can exceed $90 \%$ during a HiPIMS discharge, ${ }^{12}$ full ionization may not be required, or even desirable in all cases. This can be illustrated by the works of Aiempanakit et al. ${ }^{13}$ on $\mathrm{TiO}_{2}$, and Alami et al. ${ }^{14}$ on $\mathrm{CrN}$, see Figs. 1(a) and 1(b), respectively. (It has to be pointed out here that for the latter study, previously unpublished data points for the deposition rate have been provided by the corresponding author of Ref. 14.) By decreasing the ionization degree and approaching a DCMS-like discharge (e.g., low peak target current or peak target power) the 
deposition rate loss was reduced, while the beneficial effects on optical properties and surface roughness due to a partially ionized deposition flux were still obtained. Similar results has been reported employing the recently developed modulated pulse power technique. ${ }^{15}$ From Fig. 1 it can be seen that with a further increase in peak power or current-also entailing an increased degree of ionization in the deposition flux - the film properties were only marginally improved. The deposition rate, however, decreased continuously with an increased degree of ionization. By altering the process characteristics as shown in Fig. 1, not only changes in the degree of ionization of the deposition flux are expected, ${ }^{14}$ but also the deposition rate and ion energy distribution. ${ }^{13,16}$ It is well known that film microstructure and properties are influenced by the arrival rate of film forming species, ${ }^{17}$ as well as the abundance of ions and ion energy distribution in the deposition flux. ${ }^{18,19}$ Therefore, simultaneous alteration of the aforementioned parameters makes unambiguous understanding of fundamental mechanisms influencing films grown under different conditions difficult.

Another approach to navigate in the HiPIMS parameter space is by combining DCMS and HiPIMS in a single process. This approach has been implemented for deposition of multilayered films using two cathodes, where one was operating in DCMS mode and the other in HiPIMS mode. ${ }^{20}$ This combination provided for HiPIMS-like film properties for both types of layers. ${ }^{21}$ The combination of processes has also been demonstrated using a single cathode by superposition of DCMS and HiPIMS, ${ }^{22}$ mainly in order to ensure reliable plasma ignition and a steep current increase by providing access to charged particles at the onset of the pulse from the existing DCMS plasma. ${ }^{22,23}$ All of the aforemen-

(a)

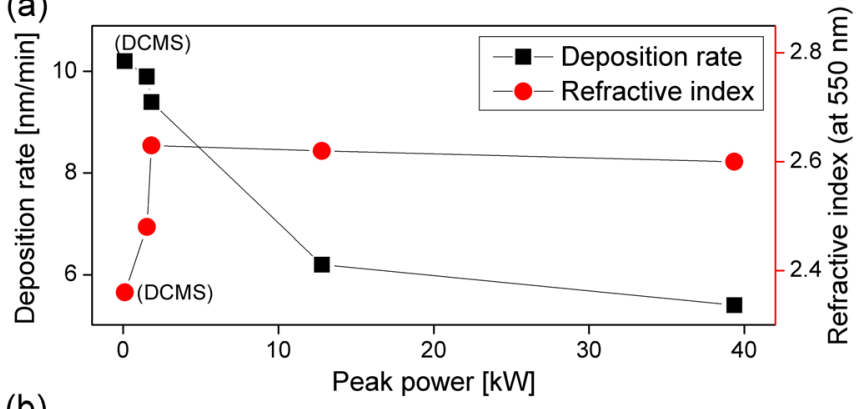

(b)

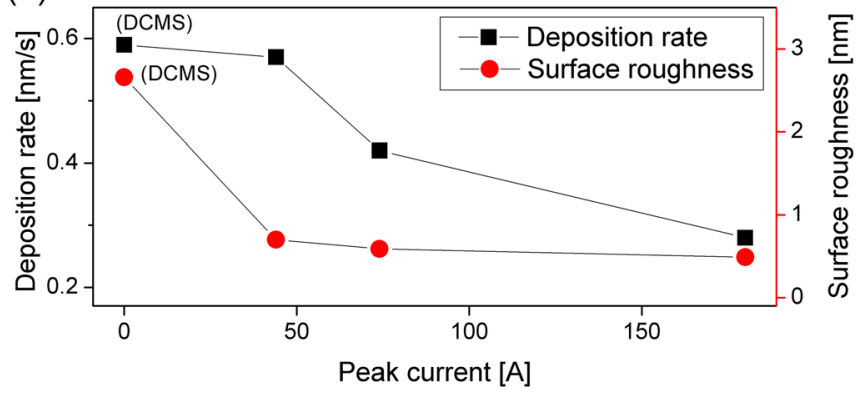

FIg. 1. (Color online) (a) Deposition rate and refractive index vs pulse peak power from Aiempanakit et al. (Ref. 13) on $\mathrm{TiO}_{2}$. and (b) deposition rate and surface roughness vs pulse peak current from Alami et al. (Ref. 14) on $\mathrm{CrN}$. tioned studies have shown that a more DCMS-like HiPIMS plasma can be advantageous from a deposition rate perspective while maintaining all, or close to all, of the advantages from a film property perspective. However, the degree of ionization itself, and its influence on film properties, has not been isolated since in all the above-discussed studies the deposition rates and/or ion energies were varied along with the degree of ionization.

In the present work, DCMS and HiPIMS, superimposed on a single cathode, are employed to alter the time averaged ion content of the deposition flux. At the same time, the ion energy distribution of the ionized sputtered metal originating from the HiPIMS process, and average deposition rate is kept constant. This is realized by keeping the HiPIMS pulse characteristics constant while altering the pulse repetition frequency to adjust the supplied HiPIMS power. A DCMS component is added for each choice of HiPIMS frequency to ensure the same average deposition rates in all experiments. We implement this strategy in order to systematically investigate the relationship between the fraction of sputtered flux generated by HiPIMS and film properties, using $\mathrm{Cr}$ as a model system.

\section{EXPERIMENTAL DETAILS}

All depositions were performed in an industrial high vacuum deposition system (Inline Coater 400, Impact Coatings ${ }^{24}$ ). Prior to the depositions the chamber was evacuated to a pressure below $4 \times 10^{-4} \mathrm{~Pa}(3 \mu$ Torr $)$. The films were deposited on $\mathrm{Si}$ substrates at an Ar pressure of $0.67 \mathrm{~Pa}$ (5 mTorr). No intentional substrate heating was used, whereas all the substrates were biased to $-50 \mathrm{~V}$. The magnetron source was equipped with a rectangular $\left(21 \times 10 \mathrm{~cm}^{2}\right) \mathrm{Cr}$ target. Two power supplies were connected to the cathode; a DCMS supply (ADL GX100/800) operating in constant current mode, and a HiPIMS supply (Sinex 3, Chemfilt AB). In order to protect the DCMS power supply from the HiPIMS transients, and allow stable DCMS operation, a diode was mounted in series with the DCMS power cable. This diode arrangement also enabled the DCMS supply to rapidly resume the set power once a HiPIMS pulse ended. As discussed in Sec. I, the HiPIMS pulse characteristics (for a single pulse), i.e., the peak values for power and current, as well as the pulse shapes were matched in all experiments. Keeping the HiPIMS pulse characteristics equal for all experiments implied that alteration of the HiPIMS power fraction, and thereby the time averaged degree of ionization, had to be controlled by varying the pulse repetition frequency. The frequency was changed in steps of $50 \mathrm{~Hz}$, ranging from 50 to $350 \mathrm{~Hz}$, resulting in duty factors between $1 \%$ and $5 \%$. The time-resolved substrate bias current was measured during the HiPIMS pulses. To obtain equal deposition rates in all experiments, a DCMS component was added according to previous deposition rate calibrations. HiPIMS typically, and also in this study, yields a lower deposition rate than DCMS for the same average power. Therefore, the combination of DCMS and HiPIMS required increasingly higher total average power as the fraction of HiPIMS power increased, as seen in Table I. 
TABLE I. Deposition conditions and resulting values for the different combinations of DCMS and HiPIMS powers. To maintain the same deposition rate for all experiments the total average power was increased as the HiPIMS power fraction increased. As the HiPIMS pulse characteristics were equal for all experiments, the HiPIMS power, and thus, the degree of ionization was adjusted by changing the pulse repetition frequency.

\begin{tabular}{lccccc}
\hline \hline $\begin{array}{l}\text { Pulse } \\
\text { repetition } \\
\text { frequency } \\
(\mathrm{Hz})\end{array}$ & $\begin{array}{c}\text { Total } \\
\text { average } \\
\text { power } \\
(\mathrm{kW})\end{array}$ & $\begin{array}{c}\text { HiPIMS } \\
\text { power } \\
\text { fraction } \\
(\%)\end{array}$ & $\begin{array}{c}\text { HiPIMS } \\
\text { peak } \\
\text { power density } \\
\left(\mathrm{W} \mathrm{cm}^{-2}\right)\end{array}$ & $\begin{array}{c}\text { HiPIMS peak } \\
\text { current } \\
\text { density } \\
\left(\mathrm{mA} \mathrm{cm}^{-2}\right)\end{array}$ & $\begin{array}{c}\text { Deposition } \\
\text { rate } \\
(\mathrm{nm} / \mathrm{min})\end{array}$ \\
\hline $\mathrm{n} / \mathrm{a}$ & 0.86 & 0 & $\mathrm{n} / \mathrm{a}$ & $\mathrm{n} / \mathrm{a}$ & 83 \\
50 & 1.00 & 25 & 408 & 590 & 87 \\
100 & 1.11 & 45 & 430 & 624 & 88 \\
150 & 1.22 & 60 & 419 & 614 & 85 \\
200 & 1.29 & 77 & 430 & 624 & 77 \\
250 & 1.34 & 88 & 419 & 619 & 83 \\
300 & 1.40 & 98 & 408 & 619 & 72 \\
350 & 1.58 & 100 & 408 & 619 & 91 \\
\hline \hline
\end{tabular}

Film thicknesses were determined by cross section scanning electron microscopy (LEO 1550 Gemini). The film thickness values were averaged for at least six measurements spanning the full substrate cross sectional width. Surface roughness rms values were attained using a Dimension 3100 SPM atomic force microscope, over a $2 \times 2 \mu \mathrm{m}$ area. Amperometric experiments $^{25}$ were performed to relate the resulting current to the surface properties. The chromium films were exposed to a potential in the transpassive region where the faradaic current was dominated by the electrochemical oxidation of chromium to soluble chromate. ${ }^{26}$ In such experiments, the current can be directly related to the surface area and thereby the surface roughness. The oxidation of the films was performed for $1 \mathrm{~h}$ at room temperature (the potential was set to $1.0 \mathrm{~V}$ vs $\mathrm{Ag} / \mathrm{AgCl}$ ) using $1.0 \mathrm{M}$ sulfuric acid as the electrolyte. The data were obtained with an Autolab PGSTAT30 (EcoChemie), employing an $\mathrm{Ag} / \mathrm{AgCl}$ reference electrode and a folded platinum wire as the counter electrode. The films (working electrodes) were brought into contact with the electrolyte via press fitting to an o-ring in the side of a Kel-F-based electrochemical cell (the exposed area of the electrodes was $0.20 \mathrm{~cm}^{2}$ ). The current at the end of each experiment was used for evaluation and comparison. In-plane electrical resistivity was measured using four-pointprobe setup with a cylindrical probe head (Jendel Engineering) connected to a micro-ohm meter (Agilent 34420A). At least five resistivity measurements, from slightly different locations, were taken on each sample.

\section{RESULTS AND DISCUSSION}

To illustrate how the deposition rate efficiency alters between DCMS, HiPIMS, and any combination of the two, the power normalized deposition rates are plotted in Fig. 2. It is seen that when the HiPIMS power fraction increases, the deposition rate efficiency decreases linearly, which implies that the two processes are independent of each other during superposition. For pure HiPIMS, $60 \%$ of the pure DCMS power normalized rate is obtained. The independ-

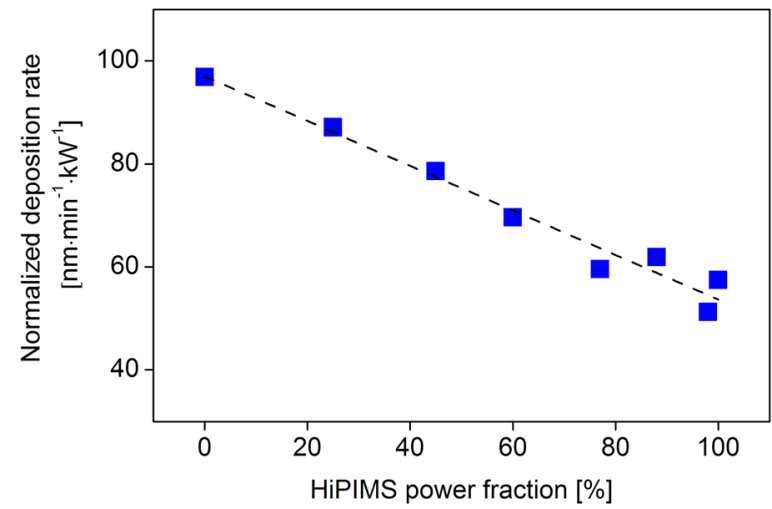

FIG. 2. (Color online) Deposition rate normalized to power as a function of the HiPIMS fraction of the total average power. Error bars are smaller than the symbols used, and the dashed line is a guide to the eye only.

ency between the two processes was valid also at the substrate position, as confirmed by measuring the time-resolved substrate bias current during the HiPIMS pulses, which showed to be insignificantly influenced by the experimental parameters. Thus, the two processes combined on a single cathode do not significantly interfere with each other with regard to deposition rate or conditions at the substrate. Since the bias current waveform for each was unaltered as the experimental conditions were varied, the time averaged bias current must increase with pulse repetition frequency. The bias current observations and the observed decrease in power normalized deposition rate, found both in the present study and in the literature, ${ }^{13}$ are consistent with an increased average degree of ionization in the deposition flux with increased pulse repetition frequency. Thus, it is here suggested that by altering the HiPIMS pulse repetition frequency, the time averaged amount of ions reaching the substrate could be varied independently of ion energy and deposition rate. Moreover, using the present experimental setup and target material we could not detect the synergy effect-where the combined DCMS and HiPIMS deposition rates superseded the sum of the parts themselves, as Bandorf et al. reported for Nichrome (Ni-Cr) and titania. ${ }^{27,28}$ Bandorf et al. observed delayed plasma ignition for pure HiPIMS, but an instantaneous current rise at the pulse onset when employing the superposition approach. This allowed for longer HiPIMS discharge time, i.e., more sputtered material and consequently higher deposition rate. Under the conditions used in the present work, the HiPIMS plasma ignites well also without the presence of a DCMS plasma preceding each pulse, which can explain the here observed absence of deposition rate synergy effect.

The microstructure of films grown using a HiPIMS power fraction of $0 \%$ (DCMS), 45\%, and $100 \%$ are illustrated by the SEM micrographs in Fig. 3. It can be seen that as the fraction of power applied by the HiPIMS power supply is increased, the microstructure of the films appears denser as the column separation is decreased and the top surface gets smoother. This trend is consistent with previous reports on morphological modifications ${ }^{14}$ and is assumed to be a consequence of increased adatom mobility ${ }^{18}$ due to an increased amount of ions in the deposition flux. From the discussion 
(a)

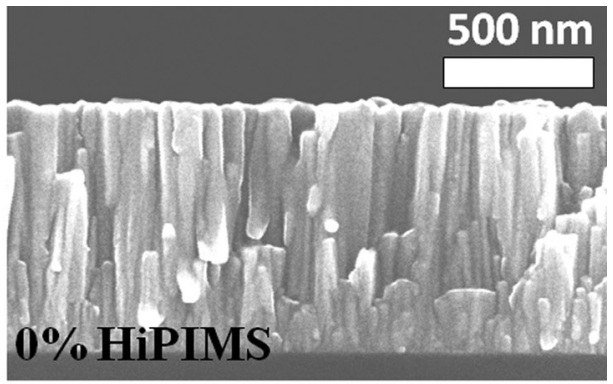

(b)

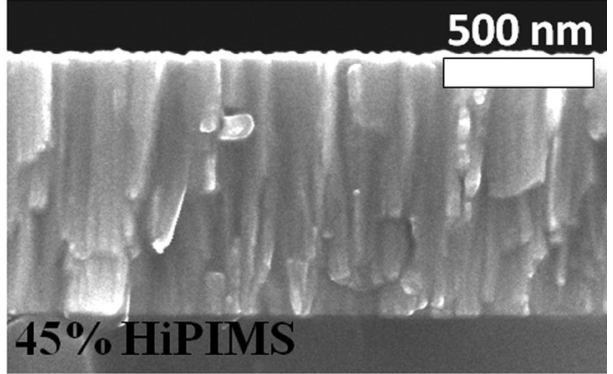

(c)

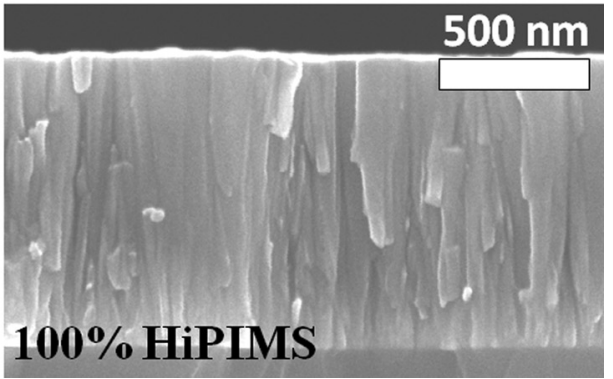

FIG. 3. SEM cross section micrographs showing the morphology for films grown using an HiPIMS power fraction of (a) $0 \%$ (DCMS), (b) $45 \%$, and (c) $100 \%$.

following Fig. 2 it was concluded that decreased power normalized deposition rate with increased HiPIMS power fraction (i.e., frequency) is consistent with an increase in degree of ionization in the average deposition flux, and in accordance, Fig. 3, confirms that the ions also arrive at the substrate in greater numbers.

The effect of the HiPIMS power fraction on roughness (rms values), electrical resistivity, and electrochemical oxidation of the $\mathrm{Cr}$ thin films is illustrated in Fig. 4. All these properties show a similar trend: after an initial overall improvement of the respective property values using a HiPIMS power fraction of up to $\sim 45 \%$, only a minor improvement is observed as a larger fraction of the total power is supplied by the HiPIMS power supply. This suggests that the influence of ionized deposition flux on the observed film properties saturates when the ionic content exceed a certain value. Decreased surface roughness, due to the ionized deposition flux, is commonly observed in studies comparing DCMS and HiPIMS, ${ }^{3,29}$ and is here consistent with the qualitative observations from the SEM, as shown in Fig. 3. The lateral resistivity will be influenced by the intercolumnar porosity, a property that, like surface roughness, is highly affected by the ionization of the deposition flux, and can thus be understood in such terms (cf. Fig. 3). For the electrochemical evaluations performed in this study, the cur-

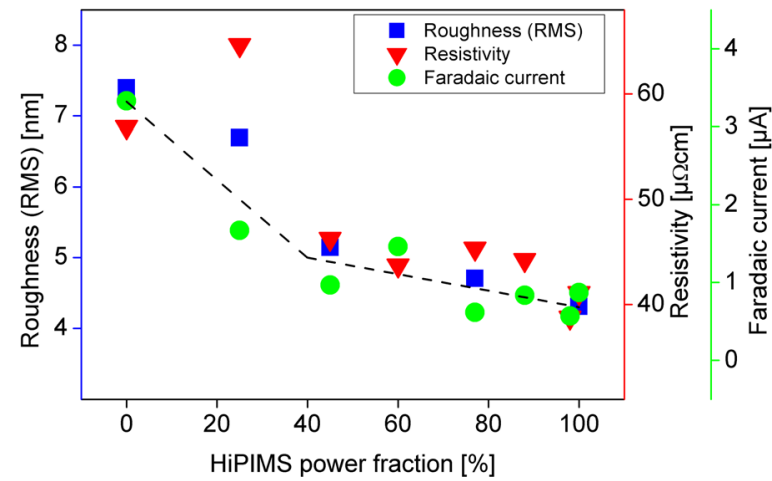

FIG. 4. (Color online) Measured film properties; roughness, electrical resistivity, and faradaic current (oxidation) as a function of HiPIMS fraction of the total average power. The dashed lines are only intended as a guide for the eye.

rent is directly related to the surface area, enabling a comparison to the surface roughness experiments. The correlation is confirmed in Fig. 4, where it is clear that the faradaic current follows the trend for the surface roughness-the films become smoother as the HIPIMS power fraction increases.

\section{SUMMARY AND CONCLUSIONS}

The results show that when combining DCMS and HiPIMS on a single cathode under the conditions used in the present experiments, the two processes can be operated independently with regard to deposition rates and ionization in the deposition flux. This allowed for tuning the amount of ions of the deposition material, keeping the deposition rate and ion energy distribution unchanged, by adjusting the DCMS and HiPIMS powers independently. For the film properties studied here-roughness, electrical resistivity, and electrochemical oxidation-a large improvement was observed already when $<40 \%$ of the average power was applied by HiPIMS, where approximately $80 \%$ of the power normalized DCMS deposition rate $\left(\mathrm{nm} \mathrm{min}{ }^{-1} \mathrm{~kW}^{-1}\right)$ was obtained. Increasing the HiPIMS power fraction resulted in marginal property improvement, but significant reduction in deposition rate efficiency.

By decoupling ionization from ion energies and average deposition rate, the results demonstrate that the degree of ionization as such is a process parameter that influences film growth. Moreover, it is qualitatively shown that there is a certain value that is sufficient for nearly full benefit with regard to film property manipulation. The strategy used in the present study also shows that degree of ionization can be isolated and controlled independently, which opens up for interesting research, e.g., regarding the role of target material ions versus process gas ions, as well as provides the possibility to tailor HiPIMS processes for specific applications with regard to ion content and deposition rate.

\section{ACKNOWLEDGMENTS}

Petter Larsson is acknowledged for hardware design, construction, and useful technical discussions. Iris Pilch is 
acknowledged for assisting with AFM measurements. M.S., U.H., and K.S. acknowledge financial support from the Swedish Research Council (VR) through Contract Nos.621-20053245, 621-2008-3222, and 623-2009-7348, respectively. F.B. would also like to thank the Swedish Foundation for Strategic Research for financial support (Strategic mobility program).

${ }^{1}$ V. Kouznetsov, K. Macák, J. M. Schneider, U. Helmersson, and I. Petrov, Surf. Coat. Technol. 122, 290 (1999).

${ }^{2}$ U. Helmersson, M. Lattemann, J. Bohlmark, A. Ehiasarian, and J. Gudmundsson, Thin Solid Films 513, 1 (2006).

${ }^{3}$ K. Sarakinos, J. Alami, and S. Konstantinidis, Surf. Coat. Technol. 204, 1661 (2010)

${ }^{4}$ J. T. Gudmundsson, Vacuum 84, 1360 (2010).

${ }^{5}$ M. Samuelsson, D. Lundin, J. Jensen, M. A. Raadu, J. T. Gudmundsson, and U. Helmersson, Surf. Coat. Technol. 205, 591 (2010).

${ }^{6}$ D. J. Christie, J. Vac. Sci. Technol. A 23, 330 (2005).

${ }^{7}$ J. Vlček, P. Kudláček, K. Burcalová, and J. Musil, J. Vac. Sci. Technol. A 25, 42 (2007).

${ }^{8}$ S. Konstantinidis, J. P. Dauchot, M. Ganciu, and M. Hecq, Appl. Phys. Lett. 88, 021501 (2006).

${ }^{9}$ D. Lundin, P. Larsson, E. Wallin, M. Lattemann, N. Brenning, and U. Helmersson, Plasma Sources Sci. Technol. 17, 035021 (2008).

${ }^{10}$ J. T. Gudmundsson, N. Brenning, D. Lundin, and U. Helmersson, J. Vac. Sci. Technol. A 30, 030801 (2012).

${ }^{11}$ J. Alami, K. Sarakinos, G. Mark, and M. Wuttig, Appl. Phys. Lett. 89, 154104 (2006).

${ }^{12}$ J. Bohlmark, J. Alami, C. Christou, A. P. Ehiasarian, and U. Helmersson, J. Vac. Sci. Technol. A 23, 18 (2005).

${ }^{13}$ M. Aiempanakit, U. Helmersson, A. Aijaz, P. Larsson, R. Magnusson, J. Jensen, and T. Kubart, Surf. Coat. Technol. 205, 4828 (2011).

${ }^{14}$ J. Alami, K. Sarakinos, F. Uslu, and M. Wuttig, J. Phys. D: Appl. Phys. 42, 015304 (2009).
${ }^{15}$ J. Lin, W. D. Sproul, J. J. Moore, Z. Wu, S. Lee, R. Chistyakov, and B. Abraham, JOM 63, 48 (2011).

${ }^{16}$ A. Hecimovic, K. Burcalova, and A. P. Ehiasarian, J. Phys. D: Appl. Phys. 41, 095203 (2008).

${ }^{17}$ C. Eisenmenger-Sittner, J. Cryst. Growth 205, 441 (1999).

${ }^{18}$ I. Petrov, F. Adibi, J. E. Greene, and J. Sundgren, Appl. Phys. Lett. 63, 36 (1993).

${ }^{19}$ A. Anders, Thin Solid Films 518, 4087 (2010).

${ }^{20}$ J. Paulitsch, P. Mayrhofer, C. Mitterer, W.-D. Münz, and M. Schenkel, Society of Vacuum Coaters 50th Annual Technical Conference Proceedings, Louisville, KY, 28 April-3 May 2007 (Society of Vacuum Coaters, Albuquerque, NM, 2007), p. 150.

${ }^{21}$ J. Paulitsch, P. Mayrhofer, W. Münz, and M. Schenkel, Thin Solid Films 517, 1239 (2008).

${ }^{22}$ M. Ganciu et al., J. Optoelectron. Adv. Mater. 7, 2481 (2005).

${ }^{23}$ R. Bandorf, S. Falkenau, V. Schmidt, and G. Mark, Society of Vacuum Coaters 50th Annual Technical Conference Proceedings, Louisville, KY, 28 April-3 May 2007 (Society of Vacuum Coaters, Albuquerque, NM, 2007), p. 477.

${ }^{24}$ H. Ljungcrantz and T. Rosell, U. S. Patent US2007/0084401 A1 (11 November 2003).

${ }^{25}$ A. J. Bard and L. R. Faulkner, Electrochemical Methods (Wiley, New York, 1980), pp. 2606-2618.

${ }^{26}$ D. Landolt, P.-F. Chauvy, and O. Zinger, Electrochim. Acta 48, 3185 (2003).

${ }^{27}$ R. Bandorf, S. Falkenau, K. Schiffmann, H. Gerdes, and U. Heckmann, Society of Vacuum Coaters 51st Annual Technical Conference Proceedings, Chicago, IL, 19-24 April 2008 (Society of Vacuum Coaters, Albuquerque, NM, 2008), pp. 317-320.

${ }^{28}$ R. Bandorf, M. Vergöhl, P. Giesel, T. Wallendorf, and G. Mark, Society of Vacuum Coaters 50th Annual Technical Conference Proceedings, Louisville, KY, 28 April-3 May 2007 (Society of Vacuum Coaters, Albuquerque, NM, 2007), p. 160.

${ }^{29}$ M. Lattemann, U. Helmersson, and J. E. Greene, Thin Solid Films 518, 5978 (2010). 